\title{
Putative cholinergic interneurons in the ventral and dorsal regions of the striatum have distinct roles in a two choice alternative association task
}

\author{
Orli Yarom $^{1}$ and Dana Cohen ${ }^{1,2 *}$ \\ Gonda Interdisciplinary Brain Research Center, Bar Ilan University, Ramat-Gan, Israel \\ 2 The Goodman Faculty of Life Sciences, Bar Ilan University, Ramat-Gan, Israel
}

\author{
Edited by: \\ James M. Tepper, Rutgers, The State \\ University of New Jersey, USA \\ Reviewed by: \\ Joshua D. Berke, University of \\ Michigan, USA \\ Mandar Shrikrishna Jog, University of \\ Western Ontario, Canada \\ *Correspondence: \\ Dana Cohen, The Gonda \\ Interdisciplinary Brain Research Center, \\ Bar Ilan University, Ramat Gan 52900, \\ Israel. \\ e-mail:danacoh@gmail.com
}

The striatum consists of GABAergic projection neurons and various types of interneurons. Despite their relative scarcity, these interneurons play a key role in information processing in the striatum. One such class of interneurons is the cholinergic tonically active neurons (TANs). In the dorsal striatum, TANs are traditionally considered to be responsive to events of motivational significance. However, in recent years, studies have suggested that TANs are not exclusively related to reward and reward-predicting stimuli, but may contribute to other processes, including responses to aversive stimuli, detecting the spatial location of stimuli and generating movement. Currently there is little data concerning TAN activity in the ventral striatum (VS) of behaving animals. Here, we simultaneously recorded neurons in the ventral and the dorsolateral (DLS) regions of the striatum while animals performed a two choice alternative association task. Our data show that a large percentage of the putative TANs in both regions responded around movement initiation and execution. The majority of these neurons exhibited directional selectivity which was stronger in DLS relative to VS. In addition, the preferred directions in VS were mostly contralateral to the recording site whereas the observed preferred directions in DLS were equally distributed contralaterally and ipsilaterally to the recording site. The most interesting difference between DLS and VS was that DLSTANs maintained activity alterations throughout the movement whereas TANs in VS exhibited short-lasting phasic activity alterations that were maintained throughout the movement by different neurons. Our findings suggest that coding of movement byTANs in both regions overlaps to some degree, yet the differences in response patterns support the notion that the TANs in DLS participate in the motor loop whereas TANs in VS convey event-related information such as movement initiation, movement direction, and end of movement.

Keywords: cholinergic interneurons, dorsal striatum, ventral striatum, motor control, chronic recording, movement coding

\section{INTRODUCTION}

The striatum, which is the main input structure of the basal ganglia, has been implicated in a variety of functions essential to shaping behavior such as goal-directed actions, action selection, habit formation, reward evaluation, flexible shifting of responses, and motor skill learning (Jog et al., 1999; Ragozzino, 2003; Costa et al., 2004; Yin et al., 2004, 2005; Lau and Glimcher, 2007; Gan et al., 2010). The striatum receives projections originating from wide portions of cortical areas (Parent and Hazrati, 1995) providing it with the information required for performing such diverse functions. Based on its input topography, the striatum has been divided into three functionally distinct regions: the dorsolateral striatum (DLS) which integrates sensorimotor information, the dorsomedial striatum (DMS), which integrates associative information and the ventral striatum (VS) which integrates limbic information (Joel and Weiner, 1994; Yin and Knowlton, 2006; Graybiel, 2008; Humphries and Prescott, 2010). The striatum consists of a relatively small number of cell types: the GABAergic projection neurons and a few types of interneurons. The projection neurons, comprising about $95 \%$ of striatal population, are the medium spiny neurons (MSNs; Gerfen, 1988). The interneurons comprise the remaining $5 \%$ and include the GABAergic fast spiking interneurons (FSIs), the tonically active neurons (TANs), and other interneurons, which have been the subject of very few studies. Despite their scarcity the FSIs and the TANs can effectively alter the activity of the projection neurons (Tepper and Bolam, 2004; Ding et al., 2010). Specifically, the TANs, presumably cholinergic interneurons (Wilson et al., 1990; Bennett and Wilson, 1999), have been studied extensively in behaving monkeys and shown to respond to events of motivational significance such as reward and stimuli predictive of reward (Kimura et al., 1984; Apicella et al., 1991, 1997; Aosaki et al., 1994; Morris et al., 2004). In recent years, studies suggest that the TANs' role may not be confined to reward and motivation but to a broader range of processes such as detection of aversive stimuli (Blazquez et al., 2002; Ravel et al., 2003; Yamada et al., 2004), recognition of the context in which motivationally significant stimuli are presented (Shimo and Hikosaka, 2001; Yamada et al., 2004; Lee et al., 2006; Ravel et al., 2006) and detection of spatial locations of stimuli (Shimo and Hikosaka, 2001; Ravel et al., 2006). A few studies have shown that TANs are 
also involved in movement control (Lee et al., 2006; Ravel et al., 2006). The vast majority of studies examining TAN activity in behaving monkeys have been conducted in the dorsal striatum, and mostly report similar responses in the caudate and putamen which are homologous to the rat DLS and DMS, respectively (but see, Yamada et al., 2004). Unlike the TANs in the DLS and the DMS, information about TAN activity in the VS is still lacking.

We addressed the role of TANs in the DLS and the VS by simultaneously recording activity of neuronal ensembles in these regions while rats performed a two choice alternative association task. We compared the nature of the TANs' response patterns observed in the two regions during movement execution to test to what extent their activations in the different regions overlap in order to decipher their specific contribution to task performance.

\section{MATERIALS AND METHODS}

All procedures were approved by Bar Ilan University institutional animal care and use committee and were performed in accordance with the National Institutes of Health guidelines.

\section{BEHAVIORAL PARADIGM}

The training procedure has previously been described in detail (Cohen and Nicolelis, 2004). Briefly, adult male Long-Evans rats were pretrained to nose poke a hole and wait for a tone $(12 \mathrm{kHz})$ to receive $50 \mu \mathrm{l}$ of water. The time delay to the tone was gradually increased until rats consistently waited for 550-700 ms during 85\% of the trials. Upon reaching criterion rats were removed from the water deprivation protocol and underwent surgery. After about 7-10 days of recovery, animals started training on the two choice alternative association task. In this stage, after nose poking the center hole for $550-700 \mathrm{~ms}$, either a high $(18 \mathrm{kHz})$ or a low $(7 \mathrm{kHz})$ tone was played and two side doors covering holes located left and right to the center hole opened. Depending on the tone played, rats had to nose poke either the left hole $(18 \mathrm{kHz})$ or the right hole $(7 \mathrm{kHz})$. After each correct trial, rats received $50 \mu \mathrm{l}$ of water as a reward in the poked hole. An error trial occurred when a rat nose poked the wrong side hole. In this case, the rat was forced to repeat the trial until performed correctly to prevent the development of a side preference. The intertrial interval changed randomly, ranging between 14 and $24 \mathrm{~s}$. An infrared source-detector system was installed behind each door, to detect the entrance and retraction of the rat's snout into and from the hole. Each session continued until at least 35 correct responses were made in each direction ( 70 correct trials). For 2 weeks rats trained one session a day during which a criterion of above $80 \%$ correct responses was reached. Although recordings were made throughout training, we report here only on the best day of training, when performance was above criterion.

\section{SURGICAL PROCEDURE}

The surgical procedure has previously been described in detail (Nicolelis et al., 1997; Jacobson et al., 2009). In brief, adult male Long-Evans rats weighing 350-450 g (Harlan and Taconic) were initially sedated by $5 \%$ isoflurane and then injected i.m. with ketamine $\mathrm{HCl}$ and xylazine $\mathrm{HCl}$ (100 and $10 \mathrm{mg} / \mathrm{kg}$, respectively). Supplementary injections of ketamine and xylazine were given as required. The rat's head was fixed in a stereotaxic frame
(Kopf Instruments, USA). An incision was made to expose the skull surface, connective tissue was removed and the skull surface cleaned. Two craniotomies, slightly larger than the electrode arrays, were made bilaterally above the dorsolateral $(\mathrm{AP}=0.5$; $\mathrm{ML}=3.9$; Depth $=3.9$ ) and ventral striatum (nucleus accumbens; $\mathrm{AP}=-1.5 ; \mathrm{ML}=1.8 ;$ Depth $=6.9) .4 \times 4$ or $4 \times 8$ electrode arrays made with isonel coated tungsten wires (diameters of 35 or 50 microns) were slowly lowered into the brain. Electrodes were fixed in place using dental cement, leaving only the connectors exposed.

Electrode placement was confirmed histologically after performing electrolytic lesions, perfusion with $4 \%$ formalin, and brain fixation with $20 \%$ sucrose, formalin, cryostat sectioning with $60 \mu \mathrm{m}$ slice thickness, and cresyl violet staining.

\section{DATA COLLECTION}

Neural activity was amplified, band-pass filtered at $150-8000 \mathrm{~Hz}$, and sampled at $40 \mathrm{kHz}$ using a multichannel acquisition processor system (MAP system; Plexon Inc, Dallas, TX, USA). The activity was initially sorted online; however, all waveforms exceeding a selected threshold were saved for later analysis. Offline sorting was done on all recorded units to ensure that only single units were used in this study.

\section{NEURONAL CLASSIFICATION}

Neurons were classified using three parameters: firing rate, coefficient of variation (cv), and valley to amplitude ratio (VAR). Firing rate was calculated as number of recorded spikes divided by session duration. The $\mathrm{cv}$ was calculated by dividing the standard deviation of the inter spike intervals (ISIs) by their mean. The VAR was calculated as the absolute value of the first valley in the waveform divided by the difference between its minimum value and the following maximum (see inset in Figure 2A).

The percent of time with ISIs $>2 \mathrm{~s}$ was calculated as in (SchmitzerTorbert and Redish, 2008; Gage et al., 2010) by summing over ISIs longer than $2 \mathrm{~s}$ and dividing the sum by the total recording time.

\section{DATA ANALYSIS \\ Movement-related neurons}

Neurons were considered movement related only if the following two conditions were met: (1) their firing rate during left or right movements was significantly different from their baseline firing rate measured two seconds prior to trial initiation; and (2) their firing rate during left or right movements was significantly different from their firing rate during the waiting period in which rats nose poked the center hole and waited to hear the played tone without moving. Firing rates were compared using the Wilcoxon signed rank test, with a criterion of $p<0.01$.

\section{Direction selective units}

Movements were divided into three phases: beginning, middle, and end. Movement-related neurons were tested for directional selectivity by testing for significant differences in their firing rate during left and right movements during each of these phases using the Wilcoxon rank sum test with a criterion of $p<0.01$. If a significant difference was found in one or more phases, the unit was considered to be direction selective. 


\section{The normalized average direction selective neuron}

The preferred direction of a neuron was determined by comparing its firing rates calculated throughout the movements in each direction and averaged over trials. The direction in which the neuron exhibited a higher firing rate was termed the preferred direction. The other direction was termed the non-preferred direction. Each movement within a trial was divided into nine equal bins and the firing rate was calculated for all neurons in each of the nine bins. The firing rate in each bin was then averaged per neuron over the entire session during trials in the preferred and non-preferred directions separately. In order to examine population activity, each neuron's activity was normalized according to the bin with the highest rate. Then the firing rate of the entire population of neurons during each bin was averaged in the two directions separately.

\section{Selectivity index (SI)}

The SI of a neuron was defined by the following equation:

$$
\mathrm{SI}=\operatorname{abs}\left(\frac{\mathrm{FR}_{\mathrm{L}}-\mathrm{FR}_{\mathrm{R}}}{\mathrm{FR}_{\mathrm{L}}+\mathrm{FR}_{\mathrm{R}}}\right)
$$

$\mathrm{FR}_{\mathrm{L}}$ and $\mathrm{FR}_{\mathrm{R}}$ are the average firing rates of each neuron during left and right trials, respectively. The firing rates in all trials were calculated in $100 \mathrm{~ms}$ bins taken at the beginning, middle, and end of the movement. The final SI of a neuron was the SI averaged over the three movement phases.

\section{Trial classification}

Spike counts in $100 \mathrm{~ms}$ bins at the beginning, middle, and end of movement were calculated for all trials. Spike count histograms were constructed and normalized to generate probability distributions of spike counts for left and right movements. The probabilities of drawing a given spike count during a trial were calculated for the left and right separately, and the movement direction in that trial was determined by the larger probability of the two.

\section{RESULTS \\ RECORDING NEURAL ACTIVITY DURING THE PERFORMANCE OF AN ASSOCIATION TASK}

The performance of association tasks has been linked to activation of both the dorsal and the ventral regions of the striatum (Atallah et al., 2007) making this task ideal for studying the similarities and differences in DLS and VS ensemble activation. In the present study rats were trained on a two choice alternative association task in which they had to associate movement to the left and to the right with a high and a low pitch tone, respectively (see Materials and Methods). 12 Long-Evans male rats were trained on the task for 2 weeks during which a criterion of above $80 \%$ correct responses was met. On average, rats reached criterion after 8 days of training. The average reaction time (the time period from playing the tone until movement onset) and movement duration in the best performed session were $0.16 \pm 0.05 \mathrm{~s}$ and $0.32 \pm 0.06 \mathrm{~s}$, respectively. In this report, we include data collected during the best performed session of each rat.

We recorded neural activity in both DLS and VS using electrode arrays of 32 and 16 tungsten wires with diameters of 35 and 50 microns. An example of electrode positioning in DLS and in VS is shown in Figures 1A,B, respectively. Although the electrode tips were positioned $\pm 100 \mu \mathrm{m}$ above and below the presented planar sections, recording sites of all animals were projected and plotted on the sections with the largest number of recording sites (Figure 1C; DLS: Bregma $=-3.86$; and VS: Bregma $=-6.82$ ). Reconstruction of electrode positioning showed that the recording electrodes were confined to the DLS and VS (Figure 1C). A total of 88 and 80 wellisolated neurons were recorded during the best performed session in DLS and VS, respectively. Firing properties such as firing rate, $\mathrm{cV}$, relative quiescence periods and waveform properties such as duration, valley to peak amplitude (VPA) and VAR of the recorded neurons were closely examined. Several of these properties enabled classification of the neurons into three well-separated clusters. The best separation was obtained when the firing rates, the cvs, and the VARs were used (Figure 2A). These parameters enabled classification of the recorded neurons into three groups: putative medium spiny neurons (pMSNs), putative tonically active neurons (pTANs), and putative fast spiking interneurons (pFSIs). Firing rate better isolated the pFSIs which typically exhibit high firing rates. The cv better isolated the pMSNs which typically exhibit high values because of their phasic firing pattern. The VAR better isolated the pTANs because their long duration spikes are cut at the end of the

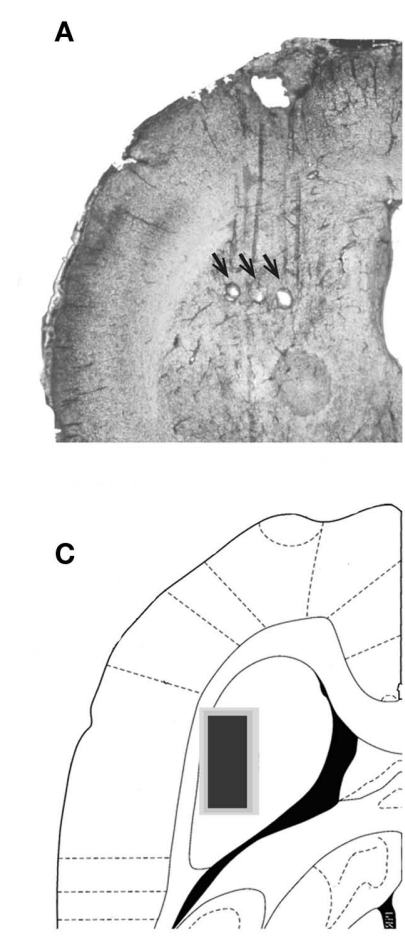

Bregma -3.86
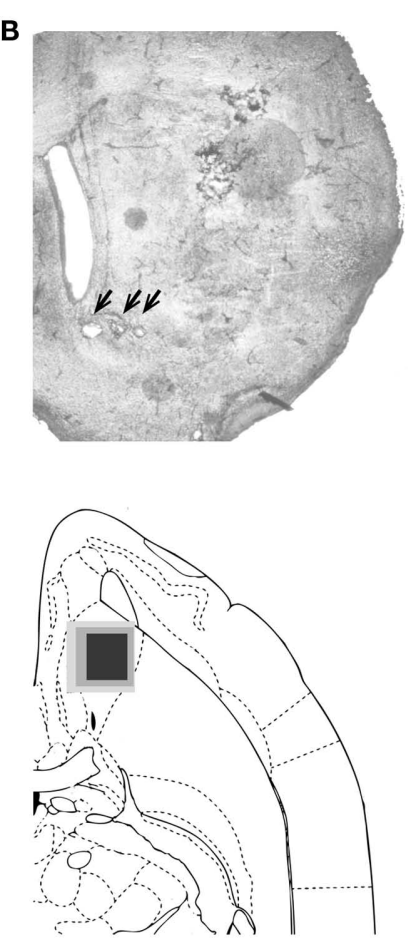

Bregma -6.82
FIGURE 1 | Recording electrodes were positioned in DLS and VS. (A,B) 60 micron sections showing electrode placement in DLS (A) and VS (B). Location of electrode tips as marked by electrolytic lesions are marked with black arrows. (C) Recording sites marked for all animals in DLS (left) and VS (right). Electrode tip positioning were superimposed on atlas planar sections at depth of -3.86 (DLS) and 6.82 (VS) from Bregma. Black marks the positioning of about $85 \%$ of the electrodes and dark and light gray mark the remaining 10 and $5 \%$, respectively. 

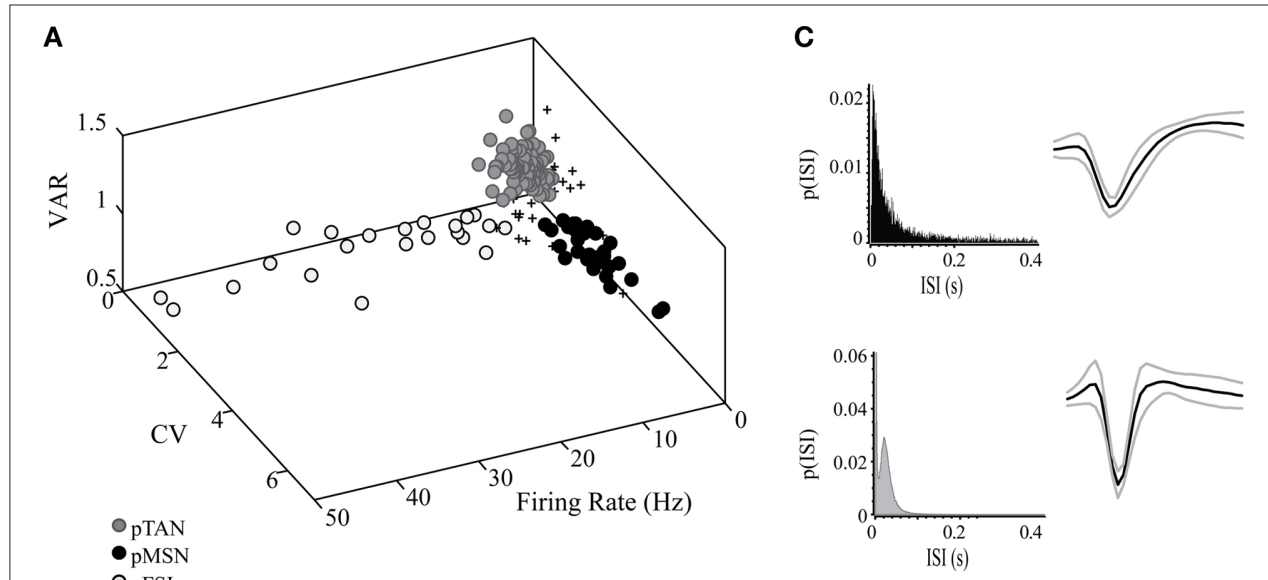

OpFSI

B
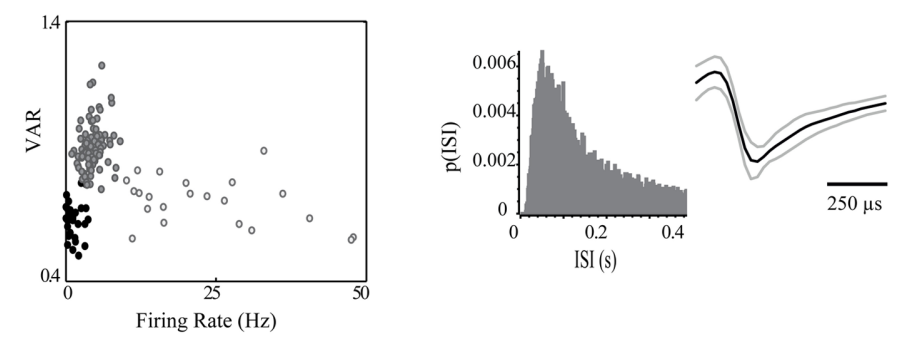

D

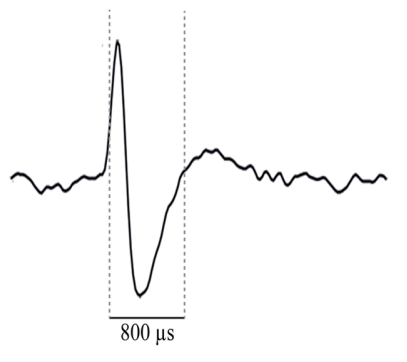

E

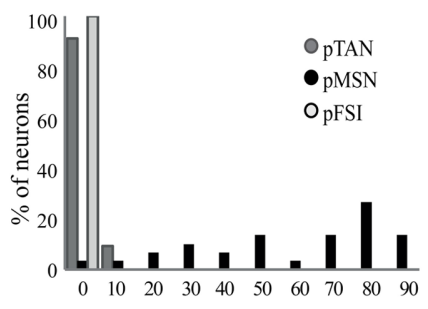

FIGURE 2 | Neurons were classified based on their firing properties and waveform shapes. (A) Scatter plot of firing rate, cV, and VAR showing the separation of the recorded neurons into three groups categorized as: pMSNs, pTANs, and pFSIs. The inset below the 3D plot demonstrates how the valley amplitude and the valley to following peak amplitude were measured. (B) A 2D plot of VAR vs. firing rate. (C) ISI distributions of three representative neurons: pMSN (top), pTAN (middle) and pFSI (bottom) and the average waveforms of each cell type (D). An example of a single pTAN recorded continuously. (E) Histograms of the percent of pMSNs, pTANs, and pFSIs with different proportions of time in ISIs longer than $2 \mathrm{~s}$. sampled segment (Figure 2D) making the valley to following peak values smaller relative to valley amplitude and consequently isolates the TANs from waveforms which are fully captured like the FSIs and the MSNs. The contribution of the VAR to the isolation of the pTANs is shown in the 2D plot in Figure 2B.

To minimize the likelihood of falsely assigning a neuron to a specific group, a set of parameters was defined for each of the groups such that if a neuron exhibited a clear separation on two of the parameters but deviated on the third, it was excluded from the analysis. Application of this strict rule excluded about $10 \%$ of the neurons. The excluded neurons are marked by a + sign in Figure 2A. The ISI probability histograms of neurons representative of the three groups and the average waveforms of all neurons within these groups are shown in Figure 2C. The pMSNs exhibited both short and long ISIs whereas the FSIs rarely exhibited ISIs longer than $200 \mathrm{~ms}$. In addition, the pTANs exhibited prolonged refractory periods and tonic firing with random ISIs. The final verification of the classification was based on the calculation of the percentage of time with ISIs longer than $2 \mathrm{~s}$ (see Materials and Methods). As expected, the vast majority of pTANs and pFSIs did not show any long pauses in firing. This contrasts with the pMSNs which were quiescent for the greater part of the recording sessions (Figure 2E).

Overall we recorded 39 and 49 pTANs in DLS and VS, respectively. The pTANs in both striatal regions exhibited similar properties. For example their firing rates were $4.87 \pm 1.63 \mathrm{~Hz}$ in DLS and $4.33 \pm 1.64 \mathrm{~Hz}$ in VS; their cvs were $1.06 \pm 0.19$ in DLS and $0.97 \pm 0.24$ in VS; their VARs were $0.92 \pm 0.07$ in DLS and
$0.92 \pm 0.10 \mathrm{in}$ VS; and the fractions of time in ISIs longer than $2 \mathrm{~s}$ were $0.02 \pm 0.03$ in DLS and $0.03 \pm 0.04$ in VS. The group of identified pTANs exhibit firing rates of about five spikes per second, cv values representing tonic firing with random distribution of long ISIs and long duration waveforms. These values are similar to those reported in primate and in rodent studies (Alexander and DeLong, 1985; Hikosaka et al., 1989; Aosaki et al., 1994; Raz et al., 1996; Joshua et al., 2008; Schmitzer-Torbert and Redish, 2008; Inokawa et al., 2010).

\section{THE pTANS IN DLS AND IN VS EXHIBIT DIFFERENTIAL DIRECTION SELECTIVITY DURING MOVEMENT}

Throughout our recordings, we observed that many of the pTANs changed their firing rates during several behavioral events compared to their basal firing rate. The neurons increased or decreased their firing rate during different parts of a trial. For example, the raster plots and peri-event time histograms (PETHs) shown in Figure 3A belong to a pTAN that increased its firing rate prior to trial initiation and during movement to the left (Figure 3A; top panel) and then decreased its firing rate during reward receipt and consumption (Figure 3A; bottom panel). It should be noted that this type of reward-related activity was more frequent in VS. We also observed that the pTANs could respond differentially during movements to the left and to the right. The pTAN in the example did not change its firing rate during movement to the right (Figure 3A; middle panel). Quantitatively, we considered a neuron to be movement related if its firing rate during movement was significantly 
A
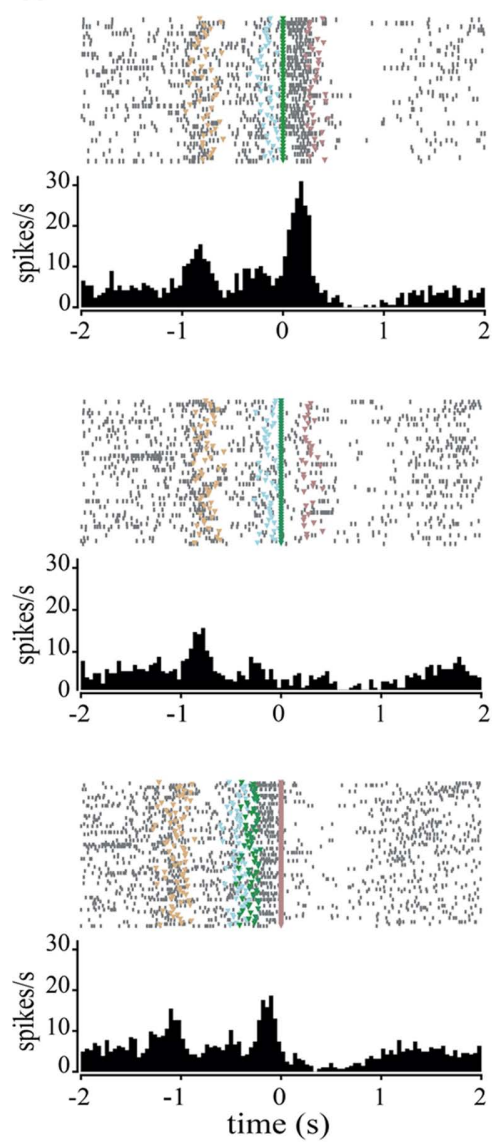

FIGURE 3 | Putative tonically active neurons in DLS and in VS exhibit movement-related activity. (A) Raster plots and their corresponding peri-event time histograms (PETHs) of a direction selective PTAN recorded in VS. Trials are presented top to bottom as they were presented during the session. Entering the center hole is marked in tan; beginning of the tone is marked in light blue; movement initiation is marked in green; and reward is
B
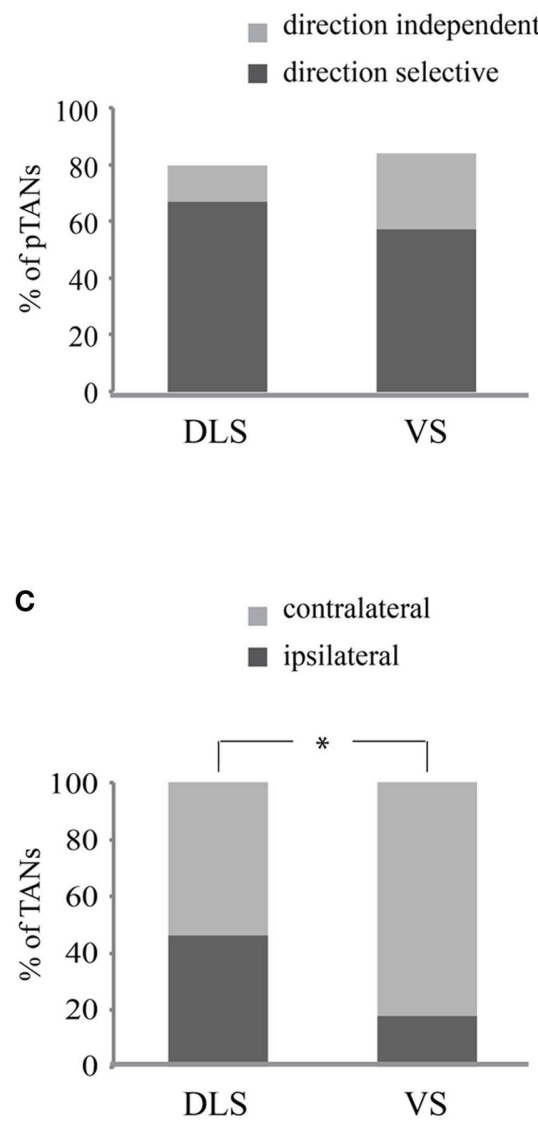

marked in violet. Top panel: plot is aligned to the beginning of movement toward the left side; middle: plot is aligned to beginning of movement to the right side; bottom: plot is aligned to reward. (B) Percent of movement related and direction selective pTANs in DLS and VS. (C) Percent of direction selective pTANs in which the preferred direction was contralateral or ipsilateral to the recording site. different $(p<0.01)$ from its firing rate during the intertrial interval and while nose poking the center hole (the waiting period) regardless of movement direction (see Materials and Methods). On the basis of these criteria, the vast majority of the recorded pTANs were found to be movement related (Figure 3B). The percentages of movement-related neurons were similar in DLS (31 out of 39; $79 \%$ ) and in VS (41 out of $49 ; 84 \% p>0.5$ ).

To test whether the pTANs are direction selective we compared the activity of movement-related neurons during left and right movements (see Materials and Methods). We observed that the majority of the pTANs were selective for movement direction (Figure 3B) and usually increased their firing rate during movement in the preferred direction while maintaining basal firing rates in the other direction or slightly decreasing it (Figure 3A; top and middle panels). The percentages of direction selective neurons were higher in the DLS (26 out of $31 ; 84 \%$ ) compared to VS (28 out of $41 ; 68 \%)$ but the difference was not significant $(p=0.13)$.

We then tested whether direction selective neurons have preferred laterality; that is, we examined whether neurons recorded in the left hemisphere responded more strongly when the animal performed a movement to the left (ipsilateral preference) or to the right (contralateral preference). Surprisingly, neurons in VS exhibited a significant preference for the contralateral direction ( $82 \%$ contralateral vs. $18 \%$ ipsilateral) whereas neurons in DLS did not exhibit a preferred laterality (54\% contralateral vs. $46 \%$ ipsilateral; $p<0.05$, Chi-square test, Figure 3C).

Overall, our recordings clearly show that pTANs in DLS and in VS exhibit significant movement-related activations with strong directional selectivity that cannot be distinguished based on the size of the responding population. However, the pTANs in VS had a strong preference for movements in directions contralateral to the recording site whereas the pTANs in DLS responded to movement in both directions regardless of recording site, which is suggestive of their different functions.

\section{DIRECTION SELECTIVE pTANs EXHIBIT DIFFERENTIAL PHASIC ACTIVATION IN DLS AND VS}

We next investigated whether the patterns of responses in DLS and VS are similar or distinct. We first examined the representative neurons in each of these regions by averaging the normalized firing 
rate of each neuron over all direction selective neurons. Despite the fact that the number of direction selective pTANs in the two striatal regions was similar, the firing patterns of the average neurons in these regions were different. In DLS the average firing rate in the preferred direction resembled a bell shape while in VS the average firing rate was concave with a slight increase toward the end of the movement (Figure 4A; top and bottom, respectively).

To pinpoint the source of this difference we calculated the number of neurons contributing selectively to the normalized response during different phases of the movement and also calculated the magnitude of the difference in firing rates during left and right movements. Based on the firing pattern of the normalized average neuron we divided the movement duration per trial into three parts: beginning, middle, and end. We compared the activity during left and right movements and tested in each part whether the firing rates in the two directions were significantly different. This allowed us to map the response of each pTAN to one of seven optional patterns depicted in Figure 4B. The observed distributions of pTAN activity patterns in DLS and VS were significantly different. Neurons in VS tended to exhibit sharp phasic responses lasting only a fraction of the movement whereas neurons in DLS tended to exhibit prolonged responses typically lasting two consecutive parts and even throughout the movement. The percent of pTANs exhibiting selectivity during a single phase was significantly higher in VS $(83 \%)$ compared to DLS $(33 \% ; p<0.01)$. To verify this result we calculated the overlap in the neuronal population during 2 consecutive phases of movement. Indeed, the overlap in neuronal activation during the beginning and middle parts of the movement was higher in DLS $(9 / 23 ; 39 \%)$ compared to VS $(3 / 21$; $14 \%)$ and reached significance when comparing the middle and end parts of the movements (DLS: $17 / 25 ; 68 \%$; VS: 5/25; 20\%; $p<0.01$ ). The observed activation patterns indicate that VS pTANs exhibit short-lasting phasic responses during different parts of the movement whereas DLS pTANs contribute throughout the movement.

To differentiate the contributions of population size and modulation strength to the normalized average neuron in both regions, we calculated selectivity indices for each of the pTAN populations. Selectivity indices averaged over DLS pTANs were significantly higher than the pTANs of VS (Figure 4C; DLS: SI $=0.57 ; n=10$; and VS: SI $=0.30 ; n=7$, Wilcoxon rank sum test, $p<0.01)$. The
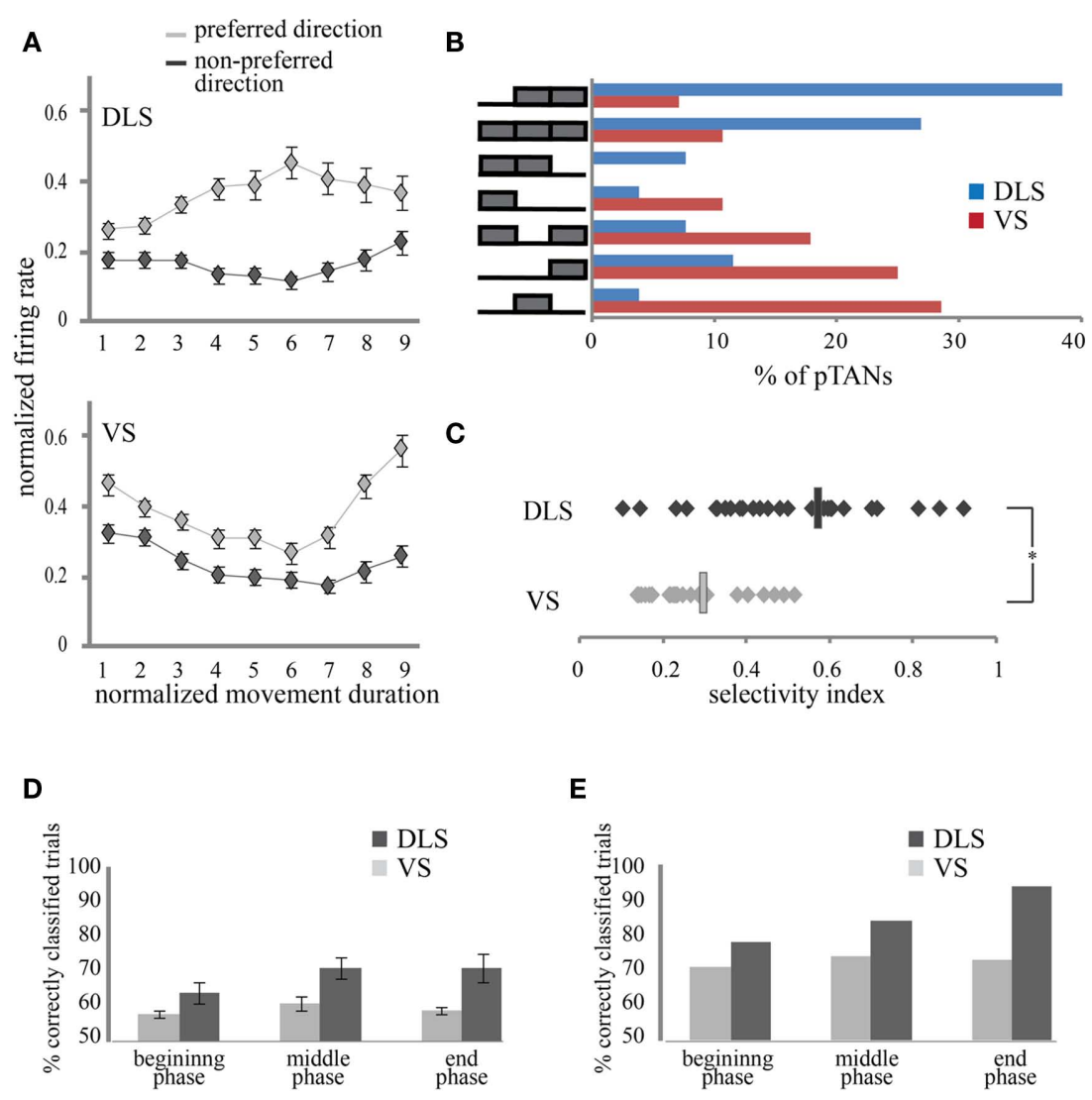

FIGURE 4 | Firing patterns characteristics of pTANS in DLS and in VS. (A) Average, normalized population activity of direction selective DLS (top) and VS (bottom) pTANs during movement, separated into preferred and non-preferred directions. Error bars are standard error of means. (B) pTANs in DLS and in VS exhibit distinct direction selectivity patterns. On the left: selectivity patterns marking movement phases with significant difference between left and right activation. For example, the top row includes neurons which exhibited significant

left and right firing differences during the middle and end of movement but not after movement initiation. On the right: the percent of pTANs in DLS (blue) and VS (red) assigned to each of the patterns. (C) Selectivity indices of direction selective pTANs in DLS and VS. Average values of SI are marked by rectangles. (D) Percent of trials in which movement direction was correctly predicted from the activity of single neurons, averaged over animals. Error bars are standard error of the means. (E) Percent of trials predicted correctly by the best neurons in each striatal region. 
differences in selectivity indices calculated for DLS and VS during the three movement phases were not significant (DLS: 0.49, 0.59, and 0.63 ; VS: $0.23,0.39$, and $0.29, p>0.1$ ). In DLS, the percent of direction selective neurons during the middle (77\%) and end $(85 \%)$ of movement was almost twice as high as that at the beginning $(46 \%)$ of the movement whereas in VS these differences were moderate $(39,46$, and $61 \%$; at the beginning, middle, and end of movement, respectively; Figure 4B). It seems that the modulation strength and the population size are synergistic in action and therefore contribute together to the observed modulation in the response of the normalized average neuron (Figure 4A). The difference in selectivity indices and population size between the two regions indicates that pTANs in DLS exhibit more pronounced activation when animals move in the preferred direction compared to pTANs in VS.

In order to further elucidate the differences in neuronal activation between DLS and VS we tested how well we could predict the movement direction of any given trial from the neuronal firing. For each neuron, the firing histograms during left and right movements were calculated and normalized. A trial was classified as a left or a right movement trial if the number of spikes released during this trial was more likely to be drawn from the normalized firing histogram of that direction excluding the trial itself (see methods). The percent of correctly classified trials was calculated for all neurons in both regions during the three movement phases. As expected from the selectivity indices, the activity of pTANs in DLS enabled a more reliable classification of the trials into left and right movements than that of pTANS in VS (DLS: $n=10 ; 64,71$, and 71\%; VS: $n=7 ; 58,61$, and 59\%; for beginning, middle, and end of movement, respectively; $p<0.01$; Figure 4D). The classification quality was similar during the different movement phases in both regions $(p>0.5)$ and in both regions, it was significantly better than chance level $(p<0.01)$. Importantly, in both regions there were neurons that were much better than the average in predicting movement direction (DLS: 80, 89, and 95\%; and VS: 71, 72, and $72 \%$; Figure $4 \mathrm{E}$ ) suggesting that this information is readily available in both regions.

We conclude that the difference in pTANs' activation in the two striatal regions results from two sources: the first is that pTANs in DLS tend to show more pronounced directional selectivity by exhibiting a stronger firing rate during movements in the preferred relative to the non-preferred direction. Secondly, the responses of pTANs in DLS are prolonged relative to those of pTANS in VS which exhibit short-lasting phasic responses. This kind of firing pattern is expected to flatten the average normalized firing because different neurons contribute during different parts of the movement, each with a smaller SI. Consequently, both regions enable extraction of information regarding movement direction that is significantly better than chance level, yet DLS provided more reliable information than VS.

\section{DISCUSSION}

In this study we simultaneously measured the neural activity of pTANs in DLS and VS while rats performed an associative task. Our data show without a doubt that the majority of the recorded pTANs in both striatal regions are movement related, and that most of them are also direction selective. In both regions, we observed similar proportions of movement related and direction selective pTANs. Nevertheless, the firing patterns of pTANs in both regions were quite different at the single cell level and the population. First, the preferred direction of pTANs in VS was typically contralateral to the recording site whereas in DLS the preferred direction of different pTANs was equally distributed ipsilaterally and contralaterally. In addition, pTANs in DLS tended to be selective throughout the movement while pTANs in VS mostly showed transient selectivity during different parts of the movement. Finally, pTANs in the DLS exhibited higher values of selectivity indices compared to pTANs in VS and consequently were better at providing information regarding movement direction at the single trial level.

The significant movement-related TAN activity we observed in our study challenges traditional assumptions concerning the role of TANs in reward and motivation. A few studies have reported TAN responses related to movement that seem to concur with our results (Lee et al., 2006; Ravel et al., 2006). In Lee et al. (2006) the percent of TANs that responded to movement reached $74 \%$ when movement was self timed by the animal, compared to less than $40 \%$ when movement was cued. The majority of studies in nonhuman primates have used behavioral paradigms which require a "go" cue for movement initiation which may reduce the likelihood of identifying movement-related TAN activation. Ravel et al. (2006) found that one-quarter of striatal TANs responded to the direction of movement and one-quarter responded to the context in which the stimulus-movement combination occurred. In our paradigm these two conditions cannot be distinguished because the number of error trials performed by the well trained animals was too low to enable reliable analysis of neuronal activity, suggesting that it is possible that some of the TANs we identified as movement-related code for movement in a specific context. Another possible explanation is the different movement requirements from behaving monkeys and behaving rats. In most studies that examine TAN activity in behaving monkeys, the subjects are restrained in their seat, their head is fixed, and the movements required of them are minimal. In our task, as in most behaving rat experiments, the rat is not restrained and is required to perform a complex movement which strongly activates the motor cortex (Cohen and Nicolelis, 2004) and likely the thalamus, and as a result the striatum.

The MSNs in the striatum have been shown to respond strongly during motor skill learning and assuming some similarity in the afferents of different striatal cell types supports the ability of TANs in dorsal striatum to exhibit motor responses. Indeed, Nambu et al. (2002) showed that stimulation of the primary motor cortex and the supplementary motor area caused excitation in both MSNs and TANs. In addition, about $60 \%$ of TANs were activated during passive or active movement of the forelimb. Thus, it is likely that in addition to other aspects of behavior, TANs in the rat DLS, which is the homologue of the monkey putamen, is involved in passive and active movements.

Even though movement-related activity is expected in the DLS, it is quite intriguing to see such activity in VS, a structure associated mainly with reward and motivation. However, this type of activity is in line with the hypothesized role of VS as a "limbic-motor interface" as proposed by Mogenson et al. (1980). Contrary to activity in the DLS, TANs in the VS show short-lasting, phasic direction selectivity. These TANs may provide an informative signal that a 
movement was initiated and to which direction, rather than taking part directly in movement control as is the case for the DLS TANs. The primary output region of the VS is the ventral pallidum, which is considered to be a central convergence point for many structures related to reward including the orbitofrontal, prefrontal and infralimbic cortices, the amygdala, lateral hypothalamus, ventral tegmental area, etc.(Smith et al., 2009). It is possible that after an action has been selected and initiated VS conveys the information regarding the action to ventral pallidum, where integration with the action outcome occurs.

As part of the theoretically segregated limbic loop, it is still unclear how VS receives information regarding motor responses. One possibility is that this information is conveyed by the pedunculopontine nucleus ( $\mathrm{PPn}$ ) in the brainstem. The PPn plays a role in the production and control of movement (reviewed in Jenkinson et al., 2009) and has been shown to respond to sensory and motor task events (Matsumura et al., 1997; Pan and Hyland, 2005). On the other hand, the PPn also receives inputs from many reward-related structures, including the ventral pallidum (Semba and Fibiger, 1992; Mena-Segovia et al., 2004; Winn, 2006) and provides one of the strongest excitatory inputs to the ventral tegmental area and substantia nigra pars compacta in the midbrain (Scarnati et al., 1984; Blaha and Winn, 1993; Pan and Hyland, 2005; Mena-Segovia et al., 2008). It is possible that the PPn conveys task-event information to the dopamine neurons of the midbrain, and this information is in turn conveyed to the VS. Further studies are required to clarify whether the information processed by TANs in VS is motor in nature or rather specific information essential for task performance such as movement direction, movement onset etc., and to locate the circuits transferring this information to the VS.

An important issue to address is why our neuronal sampling was skewed toward TANs rather than the neurons prevalent in striatum - the MSNs. Other studies in rats that have recorded activity in the striatum using tetrodes report that the majority of the

\section{REFERENCES}

Alexander, G. E., and DeLong, M. R. (1985). Microstimulation of the primate neostriatum. II. Somatotopic organization of striatal microexcitable zones and their relation to neuronal response properties. J. Neurophysiol. 53, 1417-1430.

Aosaki, T., Tsubokawa, H., Ishida, A., Watanabe, K., Graybiel, A. M., and Kimura, M. (1994). Responses of tonically active neurons in the primates striatum undergo systematic changes during behavioral sensorimotor conditioning. J. Neurosci. 14, 3969-3984.

Apicella, P., Legallet, E., and Trouche, E. (1997). Responses of tonically discharging neurons in the monkey striatum to primary rewards delivered during different behavioral states. Exp. Brain Res. 116, 456-466.

Apicella, P., Ljungberg, T., Scarnati, E., and Schultz, W. (1991). Responses to reward in monkey dorsal and ventral striatum. Exp. Brain Res. 85, 491-500.

recorded neurons were MSNs as expected from their prevalence in the striatum. Although the source of this misrepresentation is not clear, it is possible that the wire diameter used in our recording electrodes may have been a contributing factor. The 50 micron wires enable a larger detection volume which increases the likelihood of detecting large currents generated by distant large neurons like the TANs while losing the sensitivity required for detecting small currents generated by small cells like the MSNs and the FSIs. On the other hand, when recording neural activity in mice using microwires of 35 and 50 microns in diameter, the misrepresentation observed in rats disappear (unpublished data and see also Costa et al., 2006; Yin et al., 2009). Further studies have to be performed in order to clarify this issue.

\section{CONCLUSION}

The differences we observed in the firing patterns of TANs in DLS and in VS support a distinct role for this type of interneuron in each striatal region. TANs in the DLS mostly exhibited directional selectivity that continued throughout the movement duration, peaking around halfway, reminiscent of movement kinematics for ballistic movements. It is possible, therefore, that these interneurons participate in the motor loop and are involved in real-time control of movements. The pTANs in VS, on the other hand, showed short-lasting transient directional selectivity that was maintained throughout the movement by different neurons. Consequently, these neurons better fit to provide information regarding different movement-related events such as movement direction, movement initiation and expectation of movement ending. Our study calls for reassessment of the TANs' role in motor processing using a variety of behavioral paradigms.

\section{ACKNOWLEDGMENTS}

This study was supported by the Israel Science Foundation (ISF) grant \# 861/06.

Costa, R. M., Cohen, D., and Nicolelis, M. A. (2004). Differential corticostriatal plasticity during fast and slow motor skill learning in mice. Curr. Biol. 14, 1124-1134.

Costa, R. M., Lin, S. C., Sotnikova, T. D., Cyr, M., Gainetdinov, R. R., Caron, M. G., and Nicolelis, M. A. (2006). Rapid alterations in corticostriatal ensemble coordination during acute dopaminedependent motor dysfunction. Neuron 52, 359-369.

Ding, J. B., Guzman, J. N., Peterson, J. D., Goldberg, J. A., and Surmeier, D. J. (2010). Thalamic gating of corticostriatal signaling by cholinergic interneurons. Neuron 67, 294-307.

Gage, G. J., Stoetzner, C. R., Wiltschko, A. B., and Berke, J. D. (2010). Selective activation of striatal fast-spiking interneurons during choice execution. Neuron 67, 466-479.

Gan, J. O., Walton, M. E., and Phillips, P. E. (2010). Dissociable cost and benefit encoding of future rewards by mesolimbic dopamine. Nat. Neurosci. 13, 25-27.

Gerfen, C. R. (1988). Synaptic organization of the striatum. J. Electron Microsc. Tech. 10, 265-281.

Graybiel, A. M. (2008). Habits, rituals, and the evaluative brain. Annu. Rev. Neurosci. 31, 359-387.

Hikosaka, O., Sakamoto, M., and Usui, S. (1989). Functional properties of monkey caudate neurons. I. Activities related to saccadic eye movements. J. Neurophysiol. 61, 780-798.

Humphries, M. D., and Prescott, T. J. (2010). The ventral basal ganglia, a selection mechanism at the crossroads of space, strategy, and reward. Prog. Neurobiol. 90, 385-417.

Inokawa, H., Yamada, H., Matsumoto, N., Muranishi, M., and Kimura, M. (2010). Juxtacellular labeling of tonically active neurons and phasically active neurons in the rat striatum. Neuroscience 168, 395-404. 
Jacobson, G. A., Lev, I., Yarom, Y., and Cohen, D. (2009). Invariant phase structure of olivo-cerebellar oscillations and its putative role in temporal pattern generation. Proc. Natl. Acad. Sci. U.S.A. 106, 3579-3584.

Jenkinson, N., Nandi, D., Muthusamy, K., Ray, N. J., Gregory, R., Stein, J. F., and Aziz, T. Z. (2009). Anatomy, physiology, and pathophysiology of the pedunculopontine nucleus. Mov. Disord. 24, 319-328.

Joel, D., and Weiner, I. (1994). The organization of the basal ganglia-thalamocortical circuits: open interconnected rather than closed segregated. Neuroscience 63, 363-379.

Jog, M. S., Kubota, Y., Connolly, C. I., Hillegaart, V., and Graybiel, A. M. (1999). Building neural representations of habits. Science 286, 1745-1749.

Joshua, M., Adler, A., Mitelman, R., Vaadia, E., and Bergman, H. (2008). Midbrain dopaminergic neurons and striatal cholinergic interneurons encode the difference between reward and aversive events at different epochs of probabilistic classical conditioning trials. J. Neurosci. 28, 11673-11684.

Kimura, M., Rajkowski, J., and Evarts, E. (1984). Tonically discharging putamen neurons exhibit set-dependent responses. Proc. Natl. Acad. Sci. U.S.A. 81, 4998-5001.

Lau, B., and Glimcher, P. W. (2007). Action and outcome encoding in the primate caudate nucleus. J. Neurosci. 27, 14502-14514.

Lee, I. H., Seitz, A. R., and Assad, J. A. (2006).Activity of tonically active neurons in the monkey putamen during initiation and withholding of movement. J. Neurophysiol. 95, 2391-2403.

Matsumura, M., Watanabe, K., and Ohye, C. (1997). Single-unit activity in the primate nucleus tegmenti pedunculopontinus related to voluntary arm movement. Neurosci. Res. 28, 155-165.

Mena-Segovia, J., Bolam, J. P., and Magill, P.J.(2004).Pedunculopontine nucleus and basal ganglia: distant relatives or part of the same family? Trends Neurosci. 27, 585-588.

Mena-Segovia, J., Winn, P., and Bolam, J. P. (2008). Cholinergic modulation of midbrain dopaminergic systems. Brain Res. Rev. 58, 265-271.

Mogenson, G. J., Jones, D. L., and Yim, C. Y. (1980). From motivation to action: functional interface between the limbic system and the motor system. Prog. Neurobiol. 14, 69-97.

Morris, G., Arkadir, D., Nevet, A., Vaadia, E., and Bergman, H. (2004). Coincident but distinct messages of midbrain dopamine and striatal tonically active neurons. Neuron 43, 133-143.

Nambu, A., Kaneda, K., Tokuno, H., and Takada, M. (2002). Organization of corticostriatal motor inputs in monkey putamen. J. Neurophysiol. 88, 1830-1842.

Nicolelis, M. A., Ghazanfar, A. A., Faggin, B. M., Votaw, S., and Oliveira, L. M. (1997). Reconstructing the engram: simultaneous, multisite, many single neuron recordings. Neuron 18 , 529-537.

Pan, W. X., and Hyland, B. I. (2005). Pedunculopontine tegmental nucleus controls conditioned responses of midbrain dopamine neurons in behaving rats. J. Neurosci. 25, 4725-4732.

Parent, A., and Hazrati, L. N. (1995). Functional anatomy of the basal ganglia. I. The cortico-basal gangliathalamo-cortical loop. Brain Res. Brain Res. Rev. 20, 91-127.

Ragozzino, M. E. (2003). Acetylcholine actions in the dorsomedial striatum support the flexible shifting of response patterns. Neurobiol. Learn Mem. 80, 257-267.

Ravel, S., Legallet, E., and Apicella, P. (2003). Responses of tonically active neurons in the monkey striatum discriminate between motivationally opposing stimuli. J. Neurosci. 23, 8489-8497.
Ravel, S., Sardo, P., Legallet, E., and Apicella, P. (2006). Influence of spatial information on responses of tonically active neurons in the monkey striatum. J. Neurophysiol. 95, 2975-2986.

Raz, A., Feingold, A., Zelanskaya, V., Vaadia, E., and Bergman, H. (1996). Neuronal synchronization of tonically active neurons in the striatum of normal and parkinsonian primates. $J$. Neurophysiol. 76, 2083-2088.

Scarnati, E., Campana, E., and Pacitti, C. (1984). Pedunculopontine-evoked excitation of substantia nigra neurons in the rat. Brain Res. 304, 351-361.

Schmitzer-Torbert, N. C., and Redish, A. D. (2008). Task-dependent encoding of space and events by striatal neurons is dependent on neural subtype. Neuroscience 153, 349-360.

Semba, K., and Fibiger, H. C. (1992). Afferent connections of the laterodorsal and the pedunculopontine tegmental nuclei in the rat: a retro- and antero-grade transport and immunohistochemical study. J. Comp. Neurol. 323, 387-410.

Shimo, Y., and Hikosaka, O. (2001). Role of tonically active neurons in primate caudate in reward-oriented saccadic eye movement. J. Neurosci. 21 , 7804-7814.

Smith, K. S., Tindell, A. J., Aldridge, J. W. and Berridge, K. C. (2009). Ventral pallidum roles in reward and motivation. Behav. Brain Res. 196, 155-167.

Tepper, J. M., and Bolam, J. P. (2004) Functional diversity and specificity of neostriatal interneurons. Curr. Opin. Neurobiol. 14, 685-692.

Wilson, C. J., Chang, H. T., and Kitai, S. T. (1990). Firing patterns and synaptic potentials of identified giant aspiny interneurons in the rat neostriatum. $J$. Neurosci. 10, 508-519.

Winn, P. (2006). How best to consider the structure and function of the pedunculopontine tegmental nucleus: evidence from animal studies. J. Neurol. Sci. 248, 234-250.
Yamada,H., Matsumoto, N., and Kimura, M. (2004). Tonically active neurons in the primate caudate nucleus and putamen differentially encode instructed motivational outcomes of action. $J$. Neurosci. 24, 3500-3510.

Yin, H.H., and Knowlton, B. J. (2006). The role of the basal ganglia in habit formation. Nat. Rev. Neurosci. 7, 464-476.

Yin, H. H., Knowlton, B. J., and Balleine, B. W. (2004). Lesions of dorsolateral striatum preserve outcome expectancy but disrupt habit formation in instrumental learning. Eur. J. Neurosci. 19, 181-189.

Yin, H. H., Mulcare, S. P., Hilario, M. R., Clouse, E., Holloway, T., Davis, M. I., Hansson, A. C., Lovinger, D. M., and Costa, R. M. (2009). Dynamic reorganization of striatal circuits during the acquisition and consolidation of a skill. Nat. Neurosci. 12, 333-341.

Yin, H. H., Ostlund, S. B., Knowlton, B. J., and Balleine, B.W. (2005). The role of the dorsomedial striatum in instrumental conditioning. Eur. J. Neurosci. 22, 513-523.

Conflict of Interest Statement: The authors declare that the research was conducted in the absence of any commercial or financial relationships that could be construed as a potential conflict of interest.

Received: 31 January 2011; accepted: 18 May 2011; published online: 31 May 2011. Citation: Yarom $O$ and Cohen D (2011) Putative cholinergic interneurons in the ventral and dorsal regions of the striatum have distinct roles in a two choice alternative association task. Front. Syst. Neurosci. 5:36. doi: 10.3389/fnsys.2011.00036

Copyright (C) 2011 Yarom and Cohen. This is an open-access article subject to a nonexclusive license between the authors and Frontiers Media SA, which permits use, distribution and reproduction in other forums, provided the original authors and source are credited and other Frontiers conditions are complied with. 